\title{
New Developments in Laser Dyes
}

\section{F. P. SCHÄFER}

Max-Planck-Institut für biophysikalische Chemie, Abteilung Laserphysik, D-3400 Göttingen, Fed. Rep. of Germany

Recent achievements and potential for further developments in laser dyes are discussed with emphasis on wavelength coverage, efficiency and photochemical stability. It is shown that significant progress in efficiency and photostability can only be expected from new laser dyes assembled in an unconventional way from molecular subunits.

In the past decade dye lasers have become indispensable tools for spectroscopy and many other scientific applications. In contrast to this, industrial applications of dye lasers are practically non-existent. The obvious reason is the relatively low efficiency and unsatisfactory reliability of dye lasers. Both deficiencies can be traced to the active medium i.e., the organic dyes used in dye lasers. So further progress in dye lasers for industrial applications is intimately linked to development of new dyes. This is even true for scientific applications, since spectroscopists, pleased as they are with their new tunable light sources, are nevertheless constantly looking for further extension of the present wavelength coverage of dye lasers, which can only be obtained with new dyes.

Thus, one can define three main goals for the development of new laser dyes:

1) extended wavelength coverage in the UV and IR,

2) higher efficiencies by increased quantum yield of fluorescence and higher pumplight absorption,

3) better photochemical stability.

In the following some ways and means of approaching these goals and inherent limitations of potential improvements will be discussed. 


\section{EXTENDED WAVELENGTH COVERAGE}

The ultimate wavelength limits of dye laser operation had been discussed before. ${ }^{1}$ The argument for a shortwavelength limit was that the high energy of light quanta necessary for the excitation of the smallest possible $\pi$-electron systems or $n$-electrons is higher than most bond energies in the dye molecule and would thus lead to a fast dissipation of the energy by bond-breaking and destroy the molecule. An estimate of the shortest possible wavelength of dye laser operation arrived at approximately $250 \mathrm{~nm}$. A recent Russian publication ${ }^{2}$ used another argument to derive an approximate limit of $300 \mathrm{~nm}$. These authors found that the transition moment from the first excited singlet state into the dissociation and ionization continua increased so rapidly at shorter wavelengths that the increased absorption by the excited molecule of pumplight and fluorescence would prevent dye laser action below $300 \mathrm{~nm}$. Experimental investigations in our laboratory and elsewhere ${ }^{2-6}$ trying to obtain laser action in solutions or vapors of small $\pi$-electron systems or tertiary amines using ArF-laser $(193 \mathrm{~nm})$ or $\mathrm{KrF}$-laser $(248 \mathrm{~nm})$ excitation were always unsuccessful. In several tertiary amines the rate of photoionization and photodissociation of the excited molecule by the absorption of a second pumplight photon was actually measured and found to be higher than the rate of stimulated emission. This effect by itself would not yet prevent dye laser action. However, plausible extrapolation estimating the absorption cross-section at the fluorescence band shows that in tertiary amines excited state absorption is always stronger than stimulated emission. Many molecules were tested at liquid nitrogen temperature in order to reduce the rate of radiationless transitions and increase the cross-section for stimulated emission around the peak of the fluorescence band by reducing its width. Although no dye laser emission below $300 \mathrm{~nm}$ could be obtained in this way the shortest yet wavelength of a dye laser was measured as $308.5 \mathrm{~nm}$ using a $7 \times 10^{-4} \mathrm{M}$ solution of $2,2^{\prime \prime}$-dimethyl-p-terphenyl in EPA at $77 \mathrm{~K}$ excited by a KrF-laser. ${ }^{7}$ Even though an extension of a few nanometers towards shorter wavelengths might not seem much, it can be quite valuable for spectroscopic purposes after frequencytripling into the VUV to yield a tuning range increase of several thousand wavenumbers. ${ }^{8}$ 
The determination of a long-wavelength limit for dye-laser operation $^{1}$ rests on the fact that with increasing wavelength the lowest triplet state finally is so low-lying that it becomes significantly thermally populated. The ensuing reaction of the long-lived triplet molecule with oxygen or impurities determine the thermal stability of the dye. A coarse estimate of this effect leads to a long-wavelength limit of useful (i.e., longer than one day) dye laser operation of about $1.5 \mu \mathrm{m}$. A recent publication ${ }^{9}$ described the use of solution of the thiopyrilium dye of the below formula in 1,2-dichloroethane to obtain<smiles>ClC1=C(C=Cc2cc(-c3ccccc3)[s+]cc2Cl)CCCC1=CC=C1C=C(c2ccccc2)Sc2ccccc21</smiles>

dye laser emission tunable from $1.15 \mu \mathrm{m}$ to $1.24 \mu \mathrm{m}$ when pumped by a Nd:YAG laser at $1.053 \mathrm{~nm}$. Very recently the tuning range could be extended to $1.32 \mu \mathrm{m}$ using the same dye with $\mathrm{C}_{3} \mathrm{~F}_{7} \mathrm{COO}$-anion in benzyl alcohol and pumping synchronously with a mode-locked Nd:YAG laser. The dye solution showed a remarkable stability over several weeks. ${ }^{10}$ With recent synthesis of longer-wavelength absorbing dyes applying the same principle of rigidized polymethine chains and very bulky end groups one can expect an extension of the long-wavelength range of dye-laser operation to near the expected limit of $1.5 \mu \mathrm{m}$ in the near future. ${ }^{11}$

The present situation as outlined above shows that we have almost reached the probable wavelength limits of dye laser operation spanning now more than two octaves of the electromagnetic spectrum. Further progress in extending the range of fundamental frequency generation is hardly possible. However, the high power densities available from state-of-the-art dye lasers allow non-linear optical schemes to be applied to transpose tunable coherent light generation to the middle VUV by frequency multiplication, frequency mixing and anti-Stokes-Raman-shifting and into the middle IR by differencefrequency mixing and Stokes-Raman shifting of the fundamentals. 
These non-linear optical methods will also benefit from increased efficiencies of generation of fundamentals to be discussed in the next section.

\section{HIGHER EFFICIENCIES}

In excimer-laser-pumped dye lasers energy efficiencies (defined as the number of dye laser photons divided by the number of absorbed pumplight photons) of up to $50 \%$ have been achieved. ${ }^{12}$ In ideal cases, i.e., small Stokes-shift, negligible excited-state absorption, and saturating signal flux, one can expect an asymptotic limit of nearly $100 \%$. For a calculation of overall efficiency this value has to be multiplied by the "wall-plug efficiency" of the pumping laser, which is about half a percent only in the case of excimer lasers and even lower for solid-state lasers, especially when frequency doubled or tripled, and for ion lasers.

Much better is the wall-plug efficiency of flashlamp-pumped dye lasers, which typically ranges from 0.5 to $0.8 \%$. Considering the high efficiency of commercial xenon flashlamps and quantum yields of fluorescence near unity for many laser dyes, one might wonder, why the efficiency is not much higher. This is explained by Figure 1 showing the spectral radiance of a typical xenon flashlamp at medium current loading and the absorption spectrum of rhodamine $6 \mathrm{G}$, the most

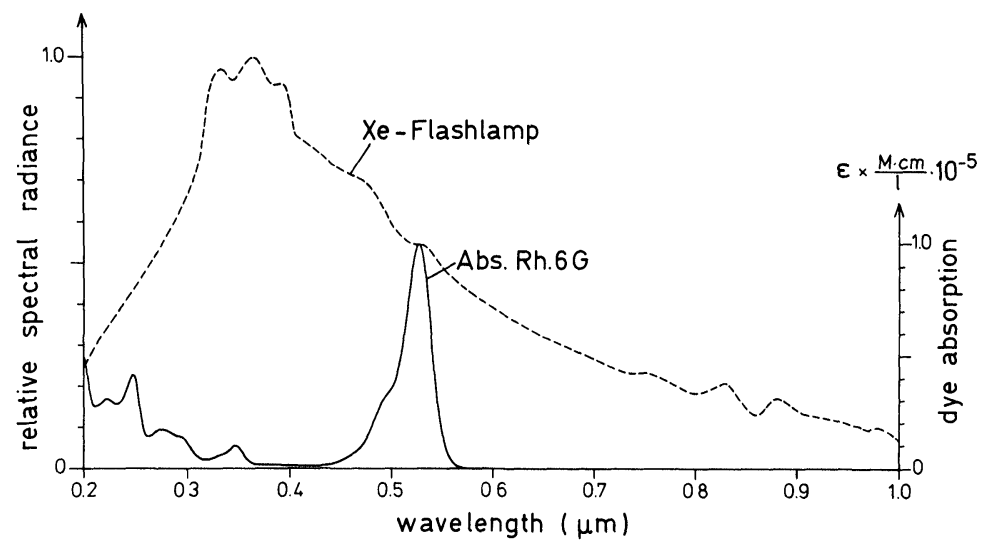

FIGURE 1 Relative spectral radiance of the typical commercial xenon flashlamp and absorption spectrum of rhodamine $6 \mathrm{G}$. 
widely used laser dye. Assuming that the concentration of the dye solution is adjusted to result in almost complete absorption of the pumplight near the peak of the absorption spectrum (as hinted by the choice of scale for emission and absorption spectra in Figure 1), one sees immediately that only a very small fraction of the pumplight is absorbed at all by the dye solution. Increasing the concentration of the dye will not help since this would result in a non-uniform distribution of excited molecules over the cross-section of the dye cell which would result in high diffraction losses and other disadvantages. A screening of absorption spectra of laser dyes reveals that no dyes are known exhibiting strong absorption over an extended region of the near UV and visible part of the spectrum. One can indeed deduce from quantum-chemical principles that the very nature of large $\pi$-electron systems essential for light absorption in the visible will always result in a relatively narrow long-wavelength absorption band joined towards shorter wavelengths by a wide region of low absorption.

Early attempts to remedy this situation made use of mixtures of dyes chosen in such a way that a shorter wavelength absorbing dye (donor) would well overlap with its fluorescence band the absorption band of a longer-wavelength absorbing laser dye (acceptor) so that radiative and/or non-radiative energy-transfer might occur from the first to the second dye. ${ }^{13}$ Most of these attempts met with little or no success. Sometimes it was even found that adding a donor decreased or completely quenched the dye laser emission.

The reason for this behavior is the fact that in these cases the energy-transfer was far from complete and left many excited molecules that would make an intersystem crossing. The ensuing strong triplet-triplet absorption would overlap with its usually broad band the fluorescence band of the laser dye and thus reabsorb partially or completely the laser emission.

One could prevent this by a nearly $100 \%$ energy transfer occurring at very high concentrations, so that the average distance between donor and acceptor molecules would be much smaller than the critical radius $\boldsymbol{R}_{0}$ for $50 \%$ radiationless energy-transfer according to Förster's theory. While this procedure can be and has been applied to some special cases of laser-pumped dye lasers, it cannot be used in the case of flashlamp-pumped dye lasers for the reasons mentioned above (e.g., high diffraction losses). 
To alleviate this problem the use of intramolecular energy transfer was proposed ${ }^{14}$ and first tried with the dye couple dimethyl-POPOP (acceptor, lasing dye) and $p$-terphenyl (donor). This dye combination was chosen for easy availability and the relative ease with which the two dye molecules could be linked by a short saturated hydrocarbon chain, even though only a relatively small increase $(\approx 30 \%)$ of absorption would mean a concomitantly small increase in laser efficiency. ${ }^{15}$ The absorption spectra of these bifluorophoric molecules showed a perfect addition of those of the two moieties, while in fluorescence only the fluorescence of the acceptor was left and no trace of the donor fluorescence could be found even in thin films of very dilute solutions. This already was an indication of $100 \%$ efficient energy transfer as was to be expected from Förster's theory for an intramolecular energy transfer with only the short distance of one to three $\mathrm{CH}_{2}$-groups between the two fluorophores. A measurement of the fluorescence risetime of the acceptor when exciting the donor with a ps pulse resulted in an energy-transfer time of less than half a picosecond, the instrumental limit. ${ }^{16}$

Lasing characteristics of these bifluorophoric dyes showed the expected efficiency increase of about $30 \%$ resulting from the increased pumplight absorption as demonstrated in Figure 2 (reproduced from ref. 15). Especially illuminating is the comparison with a mixture of the dimethyl-POPOP and $p$-terphenyl (at the same concentrations as the bifluorophoric dye) in Figure 2 which clearly shows that such a mixture gives a much lower laser output than even dimethyl-POPOP alone because of the strong $T-T$-absorption of $p$-terphenyl at the laser wavelength of $420 \mathrm{~nm}$.

The fact that these new bifluorophoric dyes still exhibit a relatively low overall laser efficiency is easily understood looking at the energy level diagram of Figure 3. While effective singlet-singlet energy transfer prevents intersystem crossing, thus eliminating $T$ - $T$-absorption by the donor moiety, the acceptor (lasing dye) moiety nevertheless will show $T-T$-absorption. For the closely related scintillator and laser dye POPOP it is known that a strong broad $T$ - $T$-absorption band overlaps its fluorescence band ${ }^{17}$ so that this is an explanation also for the low laser efficiency of dimethyl-POPOP. An early proposa ${ }^{14}$ to chemically link a triplet quencher to a laser dye to circumvent this effect by the fast depopulation of the lasing dye triplet state through intramolecular triplet-triplet energy transfer was recently realized in an elegant way. ${ }^{18}$ 


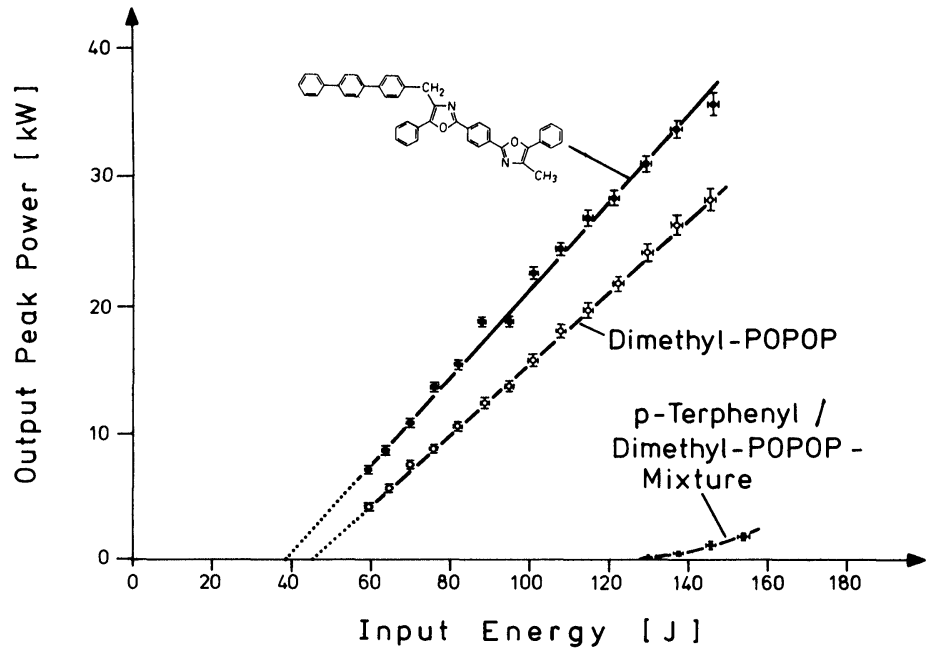

FIGURE 2 Peak laser output power versus electrical input energy of dimethylPOPOP, $p$-terphenyl- $\mathrm{CH}_{2}-\mathrm{DMP}$, and mixture of $p$-terphenyl and dimethyl-POPOP (from ref. 15).

\section{SS-Energy Transfer in Bifluorophoric Laser Dyes}

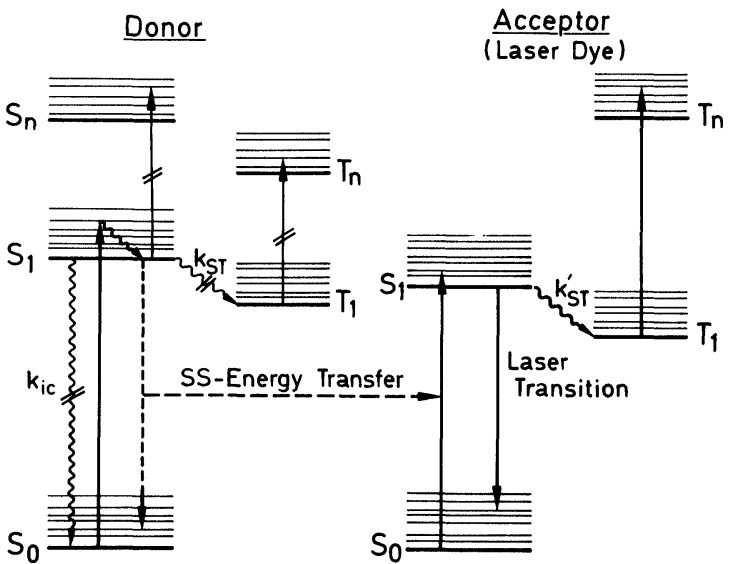

FIGURE 3 Energy level diagram for intramolecular singlet-singlet energy transfer in bifluorophoric laser dyes. 
Here again dimethyl-POPOP (DMP for short) was the lasing dye moiety, but $t$-stilbene linked to it by a short saturated hydrocarbon chain served the double purpose of donor for $S$-S-energy-transfer and acceptor for $T$ - $T$-energy-transfer as illustrated by the energylevel diagram of Figure 4. Even though the lowest triplet level of DMP was recently measured at $49 \mathrm{kcal} / \mathrm{M}^{19}$ compared to the $48 \mathrm{kcal} / \mathrm{M}$ of $T_{1}$ of $t$-stilbene, one should expect an efficient energy transfer from DMP to $t$-stilbene as shown in earlier measurements of intermolecular $T$ - $T$-energy-transfer. ${ }^{20}$

The dramatic improvement in laser efficiency realized with these dyes is shown in Figure 5 (reproduced from ref. 18), which compares the laser output energy per pulse as function of electrical input energy for DMP and the above discussed bifluorophoric dye DMP- $\mathrm{CH}_{2}-p$ terphenyl with the new bifluorophoric dyes DMP- $\mathrm{CH}_{2}-t$-stilbene and DMP- $\mathrm{CH}_{2}-\mathrm{CH}_{2}-t$-stilbene. At $50 \mathrm{~J}$ input energy, the output of the dye with a link of two $\mathrm{CH}_{2}$-groups is 33 times higher than that of DMP, and for the dye with only one $\mathrm{CH}_{2}$-group this factor is even 110 times. This already shows the strong distance dependence characteristic of $T$ - $T$-energy-transfer. A closer examination of this dependence using three dyes with different linking groups following the

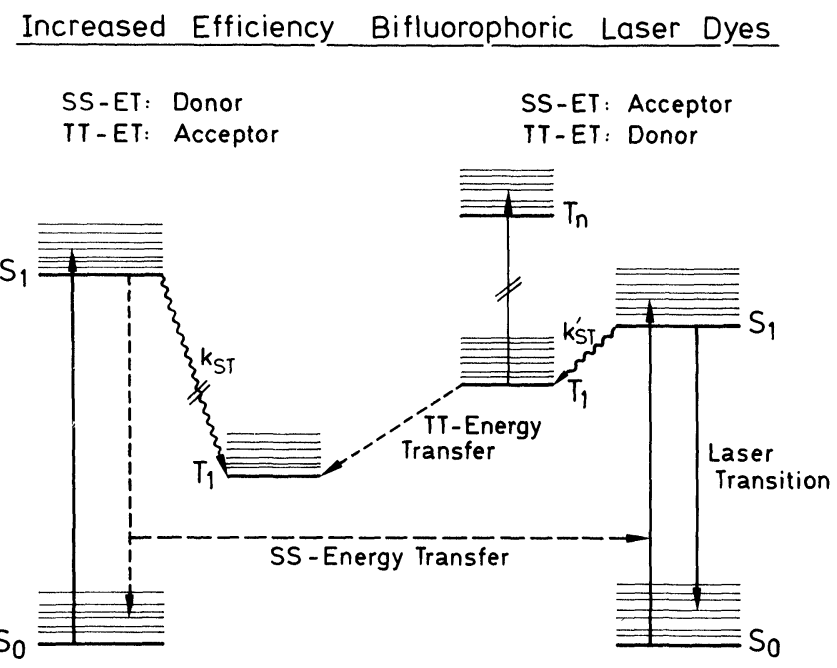

FIGURE 4 Energy level diagram of increased efficiency bifluorophoric laser dyes including intramolecular triplet-triplet energy transfer. 


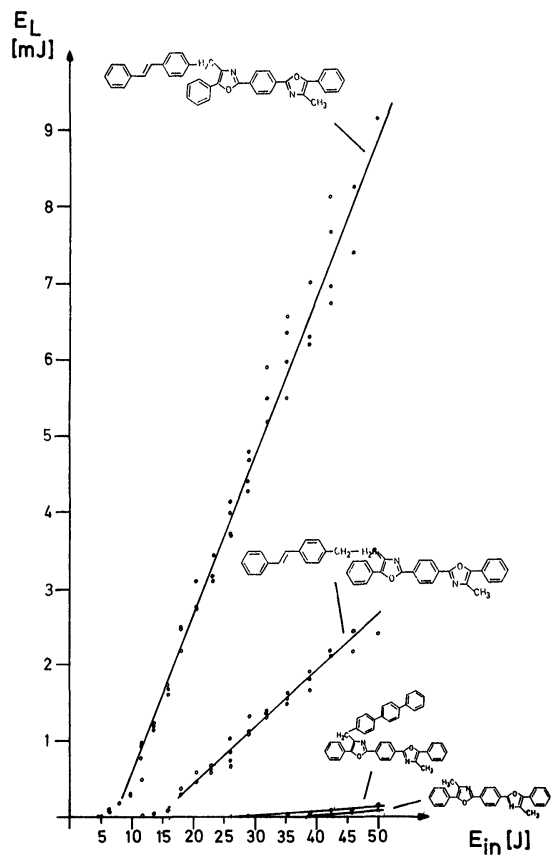

FIGURE 5 Laser pulse output energy versus electrical input energy for dimethylPOPOP and three bifluorophoric laser dyes (from ref. 18).

$T$ - $T$-absorption decay after excitation of the DMP moiety by a short $\mathrm{XeCl}$-laser pulse yielded the triplet lifetimes of the DMP moiety and the rate constants for the intramolecular $T$ - $T$-energy-transfer in these bifluorophoric dyes given in Table I. These results show convincingly

TABLE I

\begin{tabular}{|c|c|c|}
\hline Substance & $\tau_{T}(\mathrm{~ns})$ & $k_{E T}\left(\mathrm{~s}^{-1}\right)$ \\
\hline $\begin{array}{l}\text { DMP } \\
\text { DMP-CHOH-Ph-CH }-\mathrm{ST} \\
\text { DMP-CH }-\mathrm{CH}_{2}-\mathrm{ST} \\
\text { DMP-CH }-\mathrm{CH}_{2}-\mathrm{ST}\end{array}$ & $\begin{aligned} 7500 & \pm 500 \\
2600 & \pm 200 \\
70 & \pm 10 \\
7 & \pm 3\end{aligned}$ & $\begin{array}{r}(2.52 \pm 0.2) \cdot 10^{5} \\
(1.6 \pm 0.3) \cdot 10^{7} \\
(1.3 \pm 0.6) \cdot 10^{8}\end{array}$ \\
\hline
\end{tabular}

$\tau_{T}$ : triplet lifetime observed in nitrogen-purged solution in dioxane at $25^{\circ} \mathrm{C}$.

$k_{E T}$ : rate constant for triplet quenching by intramolecular energy transfer. 
that the application of intramolecular triplet quenching in laser dyes is feasible and an extremely valuable method of increasing their efficiency. A prerequisite of this method is, of course, the use of triplet quenchers that do not themselves show $T$ - $T$-absorption at the lasing wavelength and have a sufficiently short triplet lifetime to be useful at high excitation rates, when each molecule has to perform many thousands of absorption and emission acts during one laser pulse.

\section{BETTER PHOTOCHEMICAL STABILITY OF LASER DYES}

The greatest obstacle for industrial applications of dye lasers is the low photochemical stability of laser dyes. Many reports of the rapid decrease of dye laser output with prolonged operation can be found in the literature, ${ }^{21-23}$ which could be traced to photodegradation of the laser dye. Usually the decrease in dye laser concentration is less important and could, at least in principle, be compensated by the continuous addition of the right amount of new dyes to the dye circulation system. The really devastating effect is the additional absorption at the dye laser wavelength caused by the photoproducts. This was nicely shown by an investigation of the photodegradation of the laser dye 7-diethylamino-4-methylcoumarin. ${ }^{24}$ Five different photoproducts were found, two of these were the de-ethylation products with one and two hydrogen atoms respectively replacing the ethylgroups, the other three were the alcohol, aldehyde, and carboxylic acid, respectively, produced in three consecutive steps of photooxygenation with dissolved $\mathrm{O}_{2}$. The four first-mentioned photoproducts were also highly fluorescent at slightly shifted wavelengths and did not impede laser action, whereas the carboxylic acid was found to be non-fluorescent and strongly absorbing at the laser wavelength. The authors showed that the acid was preferentially adsorbed by alumina granules added to the dye reservoir of the circulation system, thus prolonging the useful lifetime of the dye solution by as much as a factor of five. The other obvious solution to make and keep the dye solution free of oxygen is often not practicable during dye laser operation and certainly too cumbersome and unreliable in harsh industrial environment. In addition, it was found with other dyes, notably rhodamine $6 \mathrm{G}$, that a slow dark reaction of dissolved oxygen 
with the photo-products partially restored laser output power, so that the presence of oxygen actually prolonged the useful lifetime of the dye solution. In such cases it was shown that first radicals were produced from the solvents by the short ultraviolet components of the pumplight or by two-photon absorption of longer-wavelength components. ${ }^{25}$ These radicals then reacted with the dye molecules to form products absorbing at the laser wavelength. This explains the strong influence of different solvents on photodegradation observed with these dyes. ${ }^{26}$ With some dyes the attack on the dye by singlet oxygen produced in a quenching reaction with laser dyes in the triplet state was observed, ${ }^{27}$ similar to many earlier observations with textile dyes.

The use of anti-oxidants, e.g., nickel-complexes, phenoles and tertiary amines, known to slow the oxidative bleaching of textile dyes, would lend itself to an improvement of photochemical stability of laser dyes, providing that these agents do not absorb at the laser wavelength nor reduce significantly the quantum yield of fluorescence of the laser dye.

Another method would be to shorten the triplet lifetime of the laser dye by intramolecular triplet quenching as discussed in the previous section. This was found to be actually so in the case of the bifluorophoric dyes with DMP and $t$-stilbene moieties. When solutions of these dyes and DMP were irradiated in the presence of air with $366 \mathrm{~nm}$ light, the relative initial rate of photochemical bleaching was 0.036 to 0.085 to 1 for DMP- $\mathrm{CH}_{2}-t$-stilbene, DMP- $\mathrm{CH}_{2}-\mathrm{CH}_{2}-t-$ stilbene, and DMP, respectively. ${ }^{27}$ Prolonged irradiation increased the rate of the bifluorophoric dyes since the trans-stilbene was partially photoisomerized to cis-stilbene, which does not show any intramolecular quenching because of its higher triplet energy of $58 \mathrm{kcal} / \mathrm{M}$. This immediately suggests the use of sterically fixed $t$ stilbene in bifluorophoric dyes to circumvent this complication. Such dyes have just been synthesized and are expected to show greatly increased photochemical stability.

Of the known quenchers of singlet oxygen the tertiary amines seem best suited for applications with laser dyes since they absorb only at wavelengths shorter than $300 \mathrm{~nm}$ and do not reduce significantly the fluorescence of at least a number of dyes. With some other dyes exciplex formation can lead to a severe drop of the quantum yield of fluorescence. 
In preliminary tests a solution of DMP in cyclohexane showed a $77 \%$ decrease of photodegradation rate when DABCO (diazabicyclooctane) was added at $5 \times 10^{-2} \mathrm{M}$ concentration. To test the effectiveness of the introduction of tertiary amino-groups as intramolecular singlet oxygen quencher diethylaminogroups were introduced into the DMP molecule replacing one hydrogen atom in one or both of the methylgroups of DMP. ${ }^{27}$ Although these substances showed an increased photodegradation rate probably due to impurities present in the raw products tested, the same addition of DABCO to these solutions as before to DMP showed a decrease of the external DABCO effect to $35 \%$ and $25 \%$ in the molecules with one and two diethylamino-groups respectively. This is a clear indication of an intramolecular singlet oxygen quenching effect of the tertiary aminogroups in these molecules. Stronger effects are to be expected from DABCO linked to DMP by a short hydrocarbon chain. Synthesis of such dyes is now under way.

\section{CONCLUSIONS}

The reported results with bifluorophoric dyes show great promise for future developments of new, unconventional laser dyes. One can envisage dyes of the type sketched in Figure 6 . These dyes would consist of a lasing dye moiety with one or more donor dye moieties linked chemically to it to increase pumplight absorption, a triplet quencher, also linked chemically to the laser dye, and perhaps even singlet oxygen quenchers and radical scavengers added in a similar way. Synthetic organic chemists who argue that such molecular monsters might be very hard to make and show low solubility and have still other difficulties should remember that the principle for the construction of new laser dyes outlined here is nothing else than the path followed by Nature so successfully in biological evolution: linking small molecular subunits to form large molecules that perform new functions not possible in a mere mixture of its constituent parts. There is strong hope that development of new laser dyes along these lines might increase efficiency and photochemical lifetime so much that many new applications, especially in industry, would start a new era in which dye lasers might play a much more important role than at present. 


\section{SCHEME FOR UNCONVENTIONAL LASER DYES}

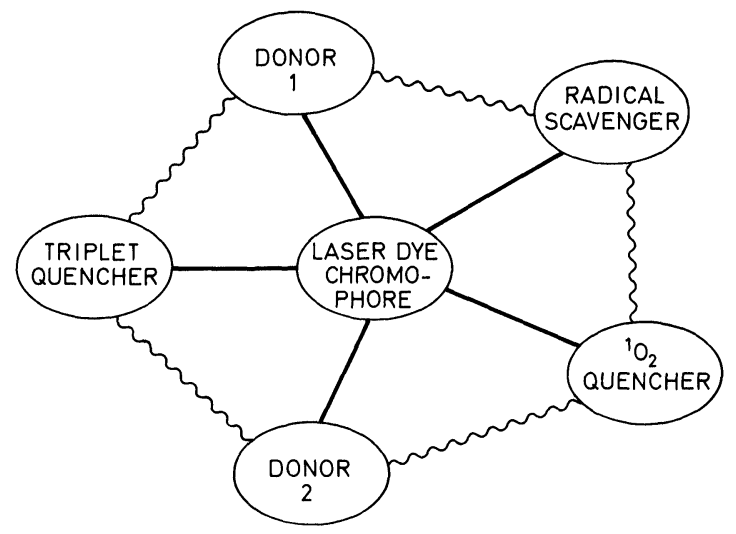

FIGURE 6 Scheme for unconventional laser dyes assembled from molecular subunits.

\section{References}

1. F. P. Schäfer (ed.), Dye Lasers, 2nd ed. (Springer, Berlin, Heidelberg, New York, 1977).

2. G. A. Abakumov, M. M. Mestechkin, V. N. Poltavets and A. P. Simonov, Sov. J. Quantum Electron. 8, 1115 (1978).

3. F.-G. Zhang, unpublished results.

4. W. Zapka and F. P. Schäfer, Appl. Phys. 20, 287 (1979).

5. A. M. Halpern, NTIS Report ADA 117439.

6. C. Rulliere and J.-C. Rayez, Appl. Phys. 11, 377 (1976).

7. F.-G. Zhang and F. P. Schäfer, Appl. Phys. B 26, 211 (1981).

8. W. Zapka, D. Cotter and U. Brackmann, Opt. Commun. 36, 79 (1981).

9. W. Kranitzky, B. Kopainsky, W. Kaiser, K. H. Drexhage and D. A. Reynolds, Opt. Commun. 36, 149 (1981).

10. B. Sens, K. H. Drexhage, A. Seilmeier and W. Kaiser, private communication.

11. K. H. Drexhage, private communication.

12. D. Basting, private communication.

13. O. G. Peterson and B. B. Snavely, British Patent No. 1255 399; W. Schmidt, W. Appt and N. Wittekindt, Z. Naturforsch. A 27, 37 (1972).

14. F. P. Schäfer, in: Tunable Lasers and Applications. eds. A. Mooradian, T. Jaeger and P. Stokseth (Springer, Berlin, 1976).

15. F. P. Schäfer, Zs. Bor, W. Lüttke and B. Liphardt, Chem. Phys. Lett. 56, 455 (1978).

16. B. Kopainsky, W. Kaiser and F. P. Schäfer, Chem. Phys. Lett. 56, 458 (1978).

17. G. Marowsky and H. Schomburg, J. Photochem. 14, 1 (1980).

18. Bodo Liphardt, Bernd Liphardt and W. Lüttke, Opt. Commun. 38, 207 (1981). 
19. Bodo Liphardt, Bernd Liphardt, W. Lüttke and D. Ouw, Appl. Phys. B 29, 73 (1982).

20. W. G. Herkstroeter and G. S. Hammond, J. Am. Chem. Soc. 88, 4769 (1966).

21. J. Jethwa, S. St. Anufrik and F. Docchio, Appl. Opt. 21, 2778 (1982).

22. A. N. Fletcher, R. H. Knipe and M. E. Pietrak, Appl. Phys. B 27, 93 (1982).

23. See Ref. 1, p. 262 for a listing of further references.

24. W. H. Winters, H. I. Mandelberg and W. B. Mohr, Appl. Phys. Lett. 25, 723 (1974).

25. I. Rosenthal, Opt. Commun. 24, 164 (1978).

26. B. A. Knyazev, S. V. Lebedev and E. P. Fokin, Sov. J. Quant. Electron. 9, 1191 (1979).

27. Bodo Liphardt, private communication. 